\title{
Generic Model for Multi-Phase Ring Oscillators
}

\author{
Paula C. Pereira ${ }^{1,2}$, Member, IEEE, António C. Pinto ${ }^{2,3}$, Member, IEEE, Luis B. Oliveira ${ }^{2,4}$, Senior Member, \\ IEEE, and Jorge R. Fernandes ${ }^{2}$, Senior Member, IEEE \\ 1 - Instituto Politécnico de Castelo Branco, Portugal \\ 2 - INESC-ID Lisboa, Instituto Superior Técnico, Universidade de Lisboa, Portugal \\ 3 - ISEL, Instituto Superior de Engenharia de Lisboa, Portugal \\ 4 - CTS-UNINOVA, DEE, FCT / Universidade Nova de Lisboa, Caparica, Portugal \\ Email: pcapereira@ipcb.pt; amcp@isel.ipl.pt; 1.oliveira@fct.unl.pt; jorge.fernandes@inesc-id.pt
}

\begin{abstract}
We present a generic model for Multiple Phase Ring Oscillators (MPRO) with $2^{n}$ phases, and derive equations for frequency, phase and phase error under the simplifying approach of injecting the error in a single element. Extensive Monte Carlo simulations, at transistor level, for the four and eight phase circuits, are in accordance with these assumptions.

These results are validated by two prototype integrated circuits, implemented in a $130 \mathrm{~nm}$ CMOS technology: The first prototype, which is a standalone four phases Ring Oscillator (RO), validates the theoretical analysis concerning the non-linear model main conclusions and, the second prototype, which is an eight phase RO, incorporated as a block of an ISM receiver, validates the generic model for CRO concept.

Index Terms - Coupled Oscillators, Multiple phase oscillators, Multiple phase ring oscillators, Oscillators, Quadrature oscillators, Ring Oscillators, Multiple Phase Clocks, Coupled Ring Oscillators.
\end{abstract}

\section{INTRODUCTION}

$\mathrm{T}$ he demand on systems for mobile communications, and for the most recent advent of digital modulations as QAM or OFDM require quadrature signals, while 8QAM, 16QAM require more clock phases (power of two) in most advanced transceivers [1].

In order to have fully integrated receivers, multiple phases are required to overcome the necessity of external high selective filters to eliminate out-of-band signals, which would increase the cost and the area for each added band. Special mixing techniques have been proposed in order to have image and harmonic frequencies rejection, which relax the RF filters specifications. Another challenge, also requiring accurate multiple-phase clocks is on control antenna arrays used for beamforming acting as a "fixed spatial filter". These multiple phase clocks have been implemented by a reference oscillator, usually at higher frequency, followed by a multiple phase clock generator $[2,3,4]$.

CMOS ROs are known for having poor phase noise performance, when compared to LC oscillators or even relaxation oscillators. However, when we couple several oscillators, the power of the output signal increase by $N^{2}(N$ is the number of interconnected oscillators), while the noise

This work was supported by national funds through FCT, Fundação para a Ciência e a Tecnologia, under project UID/CEC/50021/2013 and project UNSEEN 02/SAICT/2017. increase $N$ times, ensuring a higher $S N R$ for the system [5]. Furthermore, the coupling ensures that all the individual oscillators lock on the same frequency synchronizing them.

In [6], a general RO topology for multiple phase output and high frequency is proposed, using sub-feedback loops. This general topology also applies to even number of phases, as presented in [7]. Here, a RO topology is built with four inverters in the main ring, with feed-forward inverters between opposite nodes, which can be seen as feedback inverters to the opposite pair of inverters of the main ring. In [8] we proposed a new high-level non-linear model for MPRO, obtained used the topology presented in [7], this topology can be seen as a coupling of four RO, and the back to back inverters as a latch. Though, the high-level non-linear model presented in [8] is a relaxation oscillator based model, where the system is composed by integrators in the main loop and inverting Schmitt-triggers connected between opposite nodes. The design parameters are the integrators gain $(K)$ and the Schmitt-trigger hysteresis width $(D)$. We also derived equations for frequency, phase and phase error between phases in the presence of a mismatch, which provide interesting design guidelines for circuit implementation.

In this paper, we extrapolate the high-level non-linear model, the circuit and the equations, towards a generic model for coupled RO, with $2^{n}$ phases. With the increment of the number of coupled oscillators, a better phase noise performance, a lower frequency and more accurate phases are expected. All these trends of the extrapolated generic model led to a new eightphase oscillator and were confirmed by simulation on (C)Cadence, for the four- and eight-phase RO.

\section{GENERIC MODEL}

In [8], we derived equations for frequency, phase and phase error for a four phase (4P) ring oscillator. On equations with mismatches, we noticed the numbers " 4 " and " 3 ", and make an educated guess to extrapolate it to a higher number of phases. Equations (1) to (5), are the extrapolated equations for frequency, phase and phase error with mismatch. This

$$
f=\frac{4 K\left(K-\Delta K_{i A}\right) V_{s a t}}{K\left(D+2 V_{s a t}+\Delta D_{A}\right)+(\boldsymbol{N}-\mathbf{1})\left(K-\Delta K_{i A}\right)\left(D+2 V_{s a t}+\Delta D_{A}\right)}
$$


mismatch is applied on only one integrator $\Delta K_{I A}$ and on only one Schmitt-trigger $\Delta D_{A}$, for a $N$ phases (NP) ring oscillator.

$$
\begin{aligned}
& \Phi_{1 \_N P}=360 \cdot \frac{1+\frac{\Delta D_{A}}{\left(D+2 V_{s a t}\right)}}{N+\frac{\Delta D_{A}}{\left(D+2 V_{s a t}\right)}-(N-1) \cdot \frac{\Delta K_{i A}}{K}} \\
& \Phi_{2,3,4 \_N P}=360 \cdot \frac{1-\frac{\Delta K_{i A}}{K}}{N+\frac{\Delta D_{A}}{\left(D+2 V_{s a t}\right)}-(N-1) \cdot \frac{\Delta K_{i A}}{K}} \\
& \Delta \Phi_{1 \_N P}=270 \cdot \frac{\frac{\Delta K_{i A}}{K}+\frac{\Delta D_{A}}{\left(D+2 V_{s a t}\right)}}{N+\frac{\Delta D_{A}}{\left(D+2 V_{s a t}\right)}-(N-1) \cdot \frac{\Delta K_{i A}}{K}} \\
& \Delta \Phi_{2,3,4 \_N P}=-90 \cdot \frac{\frac{\Delta K_{i A}}{K}+\frac{\Delta D_{A}}{\left(D+2 V_{S a t}\right)}}{N+\frac{\Delta D_{A}}{\left(D+2 V_{s a t}\right)}-(N-1) \cdot \frac{\Delta K_{i A}}{K}}
\end{aligned}
$$

The extrapolated frequency equation without mismatches, for $N$ phase ring oscillator, based on equation (6), is (7).

$f_{4 P}=\frac{K V_{s a t}}{\left(D+2 V_{s a t}\right)}$

$f_{N P}=\frac{K V_{s a t}}{\frac{N}{4}\left(D+2 V_{s a t}\right)}$

Where $K$ and $D$ are defined by:

$$
\begin{aligned}
& K_{i A}=-\frac{k_{A}\left[\left(V_{D D}-V_{t}\right)\right]_{A}^{2}}{4 C_{n o d e}} \\
& D_{A}=2 \sqrt{\left(V_{D D}-V_{t}\right)^{2}+\left(\alpha^{2}+\alpha\right)+\left(V_{D D}-2 V_{t}\right)^{2}}-\left(V_{D D}-2 V_{t}\right)(1+2 \alpha) \\
& \alpha=\frac{k_{A}}{k_{B}}
\end{aligned}
$$

The non-linear model presented in [8], can be extrapolated to a higher number of phases, increasing the number of integrator blocks to $N$, however the total number of integrators must be a power of two, $N=2^{\mathrm{n}}$, and the Schmitt-trigger blocks must be connected between opposite nodes, in order to achieve the number of phases equal to the number of integrators. Fig.1 presents the non-linear model for an eight phase ring oscillator. The extrapolation at circuit level, for an eight phase ring oscillator is shown in Fig.2 [10]. The extrapolated equations for the circuit parameters $(K$ and $D)$ are the same. These equations were obtained based on the "point of view" of nodes 1 and the opposite node of node 1 .

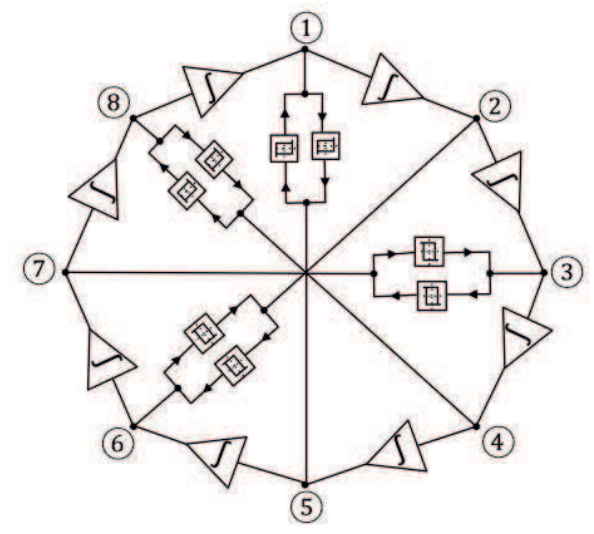

Fig. 1. Eight phase ring oscillator non-linear model.

\section{A. Discussion}

- Equation (8) show that integrator gain $K_{i A}$ depends on the transistor gain factor of the external inverter $\left(k_{A}\right)$ which is driving the node, and also on node capacitance Cnode, which are shown on Fig. 3.

-Equations (9) and (10) show that the Schmitt-trigger hysteresis width $D$, depends on a ratio between external inverter transistor gain factor, $k_{A}$, and middle inverter transistor gain factor $k_{B}$, which was denominated by $\alpha$, that can be designed.

-Considering that the inverter transistors have the same size for both the four phase ring oscillator and the eight phase ring oscillator, the comparison between equations of both topologies, lead to the following conclusions:

a) Frequency of oscillation of the four phases is two times the frequency of the eight phase ring oscillator (6) and (7) for $\mathrm{N}=8$. (See Fig. 4)

b) For the same mismatch, the frequency deviation of the four phase ring oscillator is two times the frequency deviation of the eight phase ring oscillator (1) (See Fig.6);

c) For the same mismatch, the phase error of the four phase ring oscillator is two times the phase error of the eight phase ring oscillator (4) and (5) (See Figs. 5 and 7).

Therefore, as we expected, increasing the number of phases, which is a result of increasing the number of coupled oscillators, we get an oscillator with better performance in terms of frequency and phase error robustness to mismatch, however it has the following disadvantages: decrease of the oscillation frequency, higher power consumption and higher circuit area.

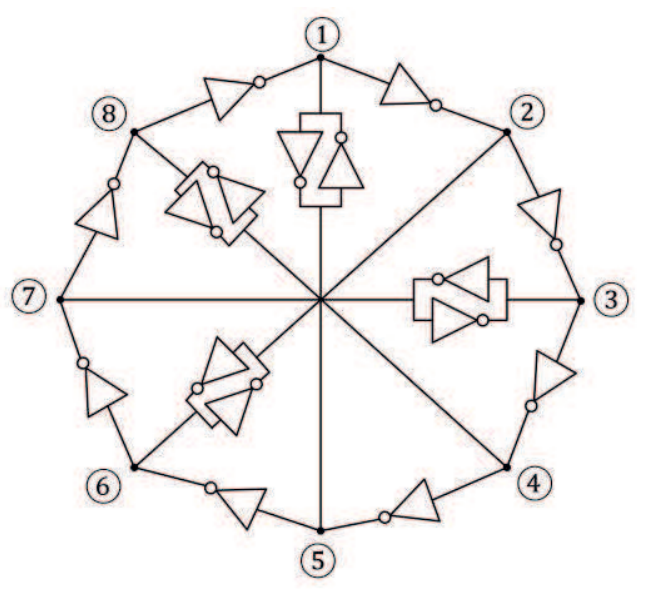

Fig. 2. Eight phase ring oscillator.

\section{Generic Model VAlidation By CirCuit Prototypes}

In this section we present two prototype circuits to validate the theoretical analysis concerning the non-linear model main conclusions (4 Phases Ring Oscillator), and to validate the generic model for coupled ring oscillator concept (8 Phases Ring Oscillator).

\section{A. 4 Phases Ring Oscillator Implemented Circuit}

In order to validate the theoretical analysis of the non-linear model, we designed a four phase ring oscillator, implemented with single-ended-starving inverters, in order to allow us to control the relationship between the external ring inverters and 


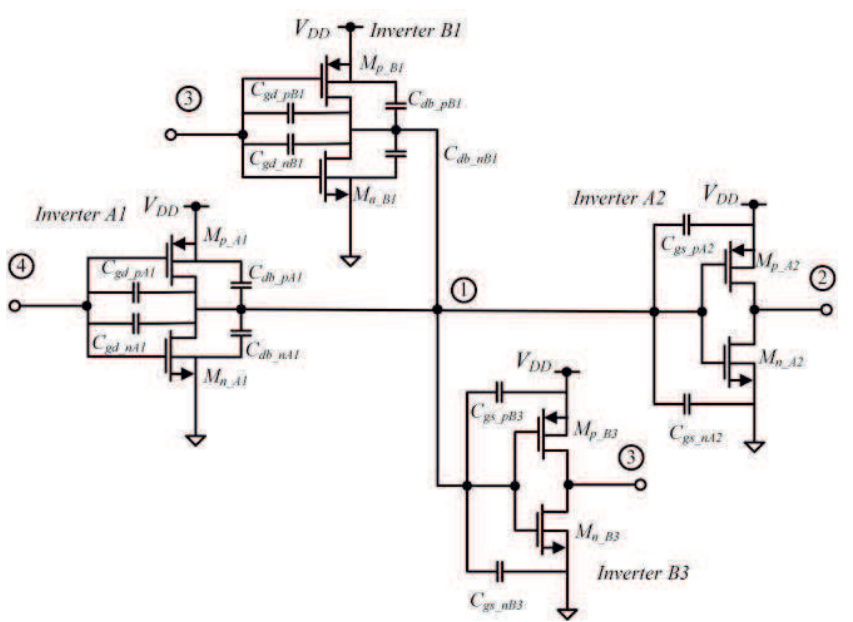

Fig. 3. Ring oscillator circuit node.

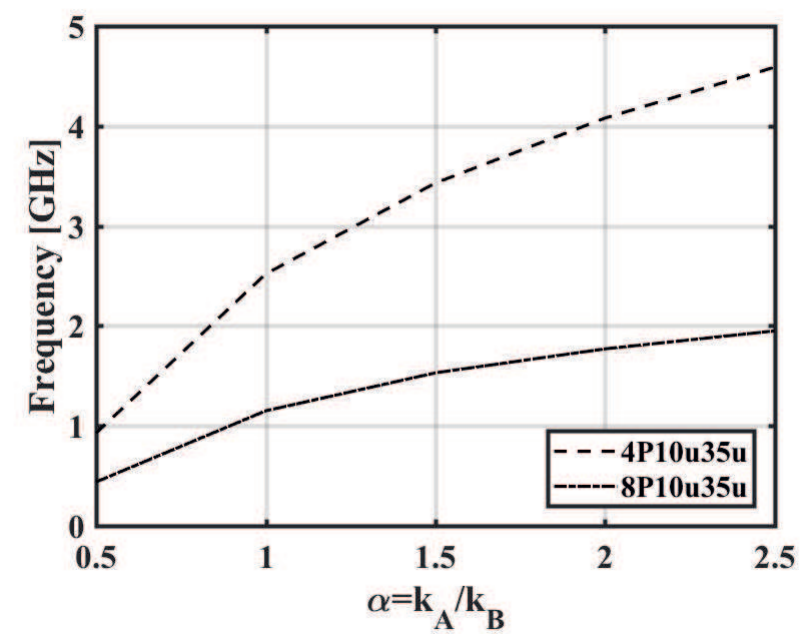

Fig.4. Frequency versus $\alpha$ factor for $4 \mathrm{P}$ and $8 \mathrm{P}$

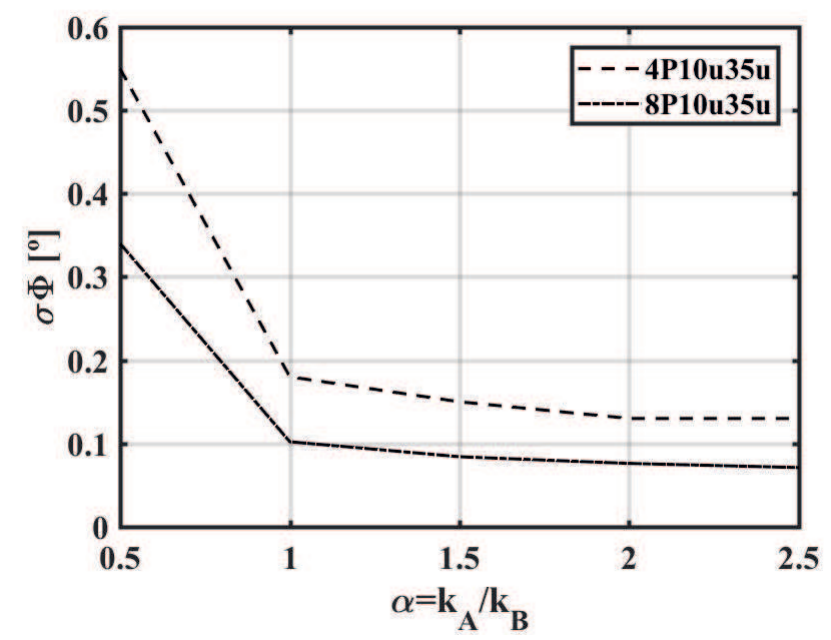

Fig. 5. Phase Error Sigma versus $\alpha$ factor for $4 \mathrm{P}$ and $8 \mathrm{P}$.

the middle inverters. All the inverters have $(35 \mu \mathrm{m} / 240 \mathrm{~nm})$ for the PMOS transistors and $(10 \mu \mathrm{m} / 240 \mathrm{~nm})$ for the NMOS. The external ring inverters and the middle inverters are connected to different current sources, being adjusted by two
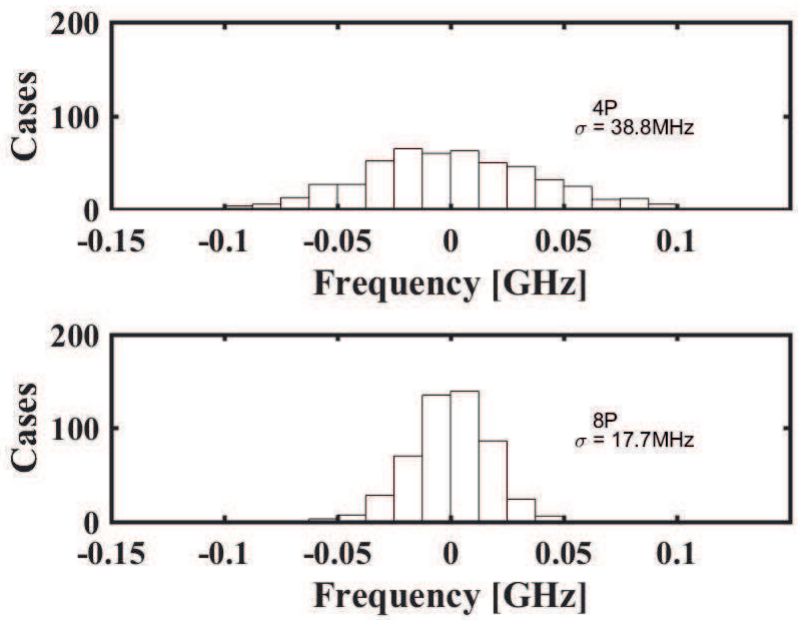

Fig. 6. Frequency Monte Carlo simulation results for 4P and $8 \mathrm{P}$
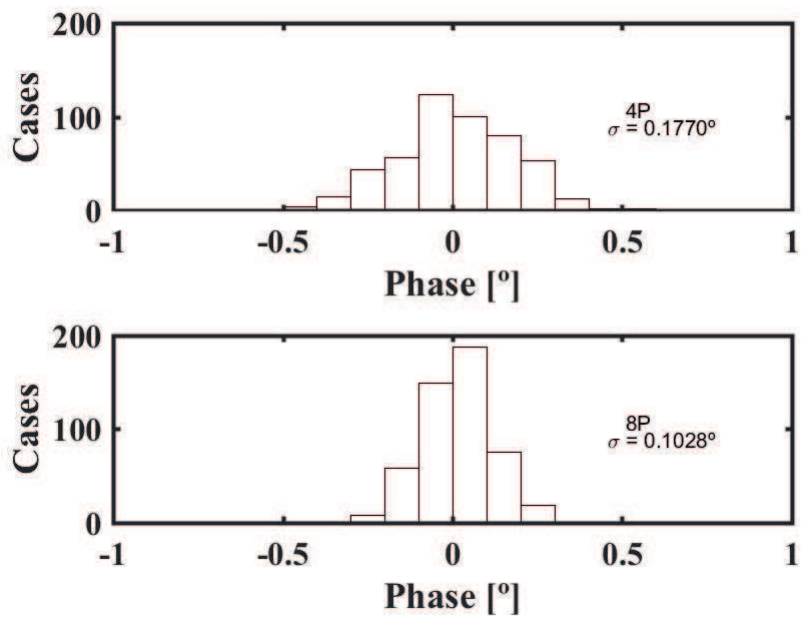

Fig. 7. Phase Monte Carlo simulation results for $4 \mathrm{P}$ and $8 \mathrm{P}$

external resistors $R_{E x t}$ and $R_{\text {Middle }}$ to simulate the variation of the relationship between the external inverters and the middle inverters. In order to compare the measurements results with theoretical results, we defined an $\alpha$ ' factor (11).

$\alpha^{\prime}=\frac{I_{\text {Ext }}}{I_{\text {Middle }}}$

Fig. 8 show the behavior of the oscillator in terms of the inverters strength, it presents frequency results for stronger external ring inverters $\left(R_{E x t}=50 \Omega ; R_{\text {Tune }}=R_{\text {Middle }}\right)$ and for stronger middle inverters $\left(R_{\text {Middle }}=50 \Omega ; R_{\text {Tune }}=R_{E x t}\right)$. There can be clearly seen, that frequency is more sensitive to external ring inverters than to middle inverters.

Fig.9 presents frequency variation with $\alpha^{\prime}$, and we can see that it has the same behavior of theoretical curve presented in Fig.4. Although, measured curve is $200 \mathrm{MHz}$ below the simulated curve.

Figs.10 presents FOM for an offset frequency of $10 \mathrm{MHz}$ (FOM@10 MHz) measurements, for stronger external inverters $\left(\alpha^{\prime} \geq 1\right)$ and for stronger middle inverters $\left(\alpha^{\prime}<1\right)$. FOM@10 MHz results, for the case the external inverters are stronger, are always above $-150 \mathrm{dBc} / \mathrm{Hz}$. There, we can also see that, FOM@10 MHz results are better when external inverters are stronger than the middle inverters, the major difference is 
$10 \mathrm{~dB}$. Figs. 9 and 10 also present simulation results for the same conditions of the measured results. Note that, the same set of $R_{\text {Ext }}$ and $R_{\text {Middle yields in different }} \alpha^{\prime}$ values. However, curves have the same behavior, but with an offset of $200 \mathrm{MHz}$ for frequency and $8 \mathrm{~dB}$ for FOM.

\section{B. 8 Phases Ring Oscillator Implemented Circuit}

The main objective of the second prototype is to validate the generic model for coupled ring oscillators concept. We design an eight phase ring oscillator, a new topology, with frequency tunable from $450 \mathrm{MHz}$ to $900 \mathrm{MHz}$. This circuit was implemented in a receiver, therefore there aren't measurements of the oscillator alone. Measurements of the receiver are presented in [10], where there are the receiver NF (Noise Figure) and gain measurements results, in terms of the local oscillator frequency (this eight phase ring oscillator). This oscillator was tested from 180 to $300 \mathrm{MHz}$. The validation of the generic model presented, is made in terms of concept.

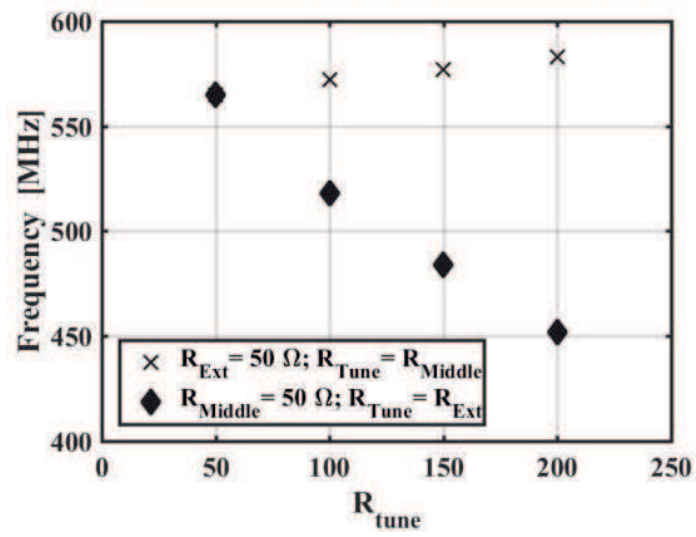

Fig. 8. Four phase ring oscillator frequency variation for stronger external inverters and for stronger middle inverters.

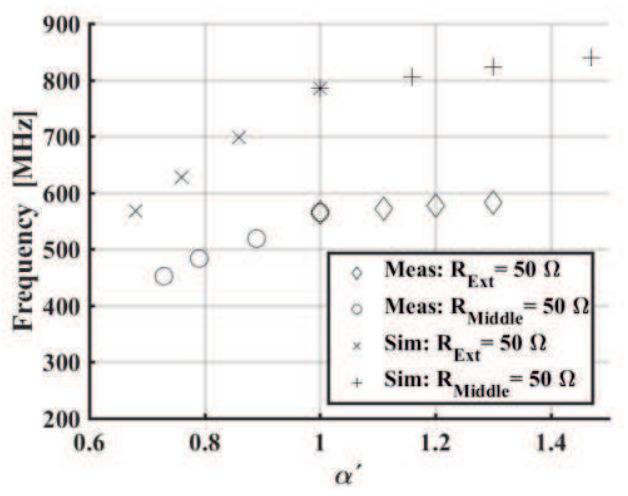

Fig. 9. Four phase ring oscillator frequency variation with $\alpha$ '.

\section{CONCLUSIONS}

A generic model for $2^{\mathrm{n}}$ phase ring oscillators is presented. Equations for frequency, phase, and phase difference error are derived, considering the introduction of mismatch in one integrator and in one Schmitt-trigger. The trends obtained with the non-linear model equations and respective circuit level equations were also confirmed with implemented circuits.

When we extrapolate from a four phase ring oscillator to an eight phase ring oscillator for the same sizing inverters, frequency gets an half value, frequency deviation and phase

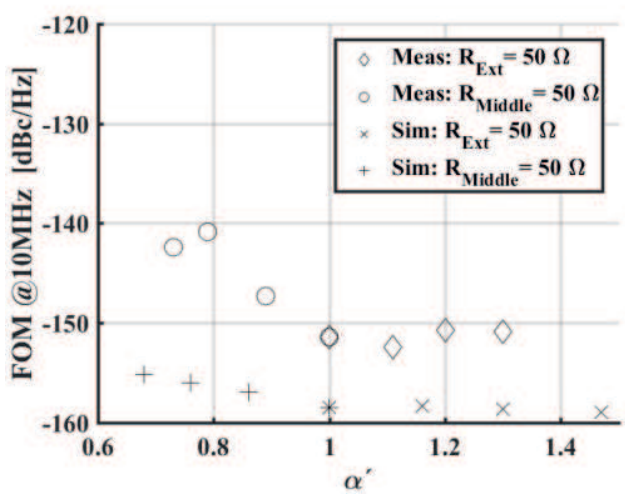

Fig. 10. Four phase ring oscillator FOM@10MHz variation with $\alpha$.

error due to mismatch are decreased to an half value, thus improving the performance in terms of phase noise and FOM, $3 \mathrm{~dB}$ for phase noise and $9 \mathrm{~dB}$ for FOM.

Independently of the number of phases, the designer must prioritize the design of the external ring inverters, because phase error due to mismatch in external ring inverters is two/three times the phase error due to mismatch in middle inverters, and frequency, is also, more sensitive to mismatch in external ring inverters. In applications where tuning is necessary, for calibration purposes for example, coarse tuning can be done using external ring inverters and fine tuning can be done using middle inverters.

\section{REFERENCES}

[1] S. Haykin, "An Introduction to Analogue and Digital Communication," John Wiley \& Sons, 1989.

[2] Z. Ru, N. A. Moseley, E. A. M. Klumperink, and B. Nauta, "Digitally Enhanced Software-Defined Radio Receiver Robust to Out-of-Band Interference," IEEE J. of Solid-State Circuits, vol. 44, no. 12, pp. 33593375, Dec. 2009, DOI: 10.1109/JSSC.2009.2032272.

[3] Z. Ru, E. A. M. Klumperink, and B. Nauta, "Discrete-Time Mixing Receiver Architecture for RF-Sampling Software-Defined Radio," IEEE J. of Solid-State Circuits, vol. 45, no. 9, pp. 1732-1745, Sep. 2010, DOI: 10.1109/JSSC.2010.2053860.

[4] K. Un, P. Mak, and R. P. Martins, "Analysis and Design of Open-Loop Multiphase Local-Oscillator Generator for Wireless Applications," IEEE Trans. Circuits Syst. I, vol. 57, no. 5, pp. 970-981, May. 2010, DOI: 10.1109/TCSI.2010.2046955.

[5] B. Razavi, "A Study of Phase Noise in CMOS Oscillators," IEEE J. of Solid-State Circuits, vol. 31, no. 3, pp. 331-343, Mar. 1996, DOI: 10.1109/4.494195.

[6] L. Sun, and T. A. Kwasniewski, "A 1.25-GHz 0.35- $\mu \mathrm{m}$ Monolithic CMOS PLL Based on a Multiphase Ring Oscillator," IEEE J. of Solid-State Circuits, vol. 36, no. 6, pp. 910-916, Jun. 2001, DOI: 10.1109/4.924853.

[7] M. Grozing, B. Phillip, and M. Berroth, "CMOS Ring Oscillator with Quadrature Outputs and $100 \mathrm{MHz}$ to $3.5 \mathrm{GHz}$ Tuning Range," in Proc. ESSCIRC, pp. 679-682

[8] P. C. Pereira, L. Oliveira and J. Fernandes, "Non-Linear Model for Multiple Phase Ring Oscilators," in Proc. PRIME, 2016, pp. 1-4.

[9] L. B. Oliveira, J. R. Fernandes, I. M. Filanovsky, C. J. M. Verhoeven and M. M. Silva, "Analysis and Design of Quadrature Oscillators," Springer Netherlands, 2008.

[10] J.R. Custodio, I. Bastos, L.B. Oliveira, J.P. Oliveira, P. Pereira, J. Goes and E. Bruun, "A $6.2 \mathrm{~mW} 0.024 \mathrm{~mm}^{2}$ Fully-Passive RF Downconverter with 12 dB Gain Enhancement using MOS Parametric Amplification," Analog Integrated Circuits and Signal Processing, vol. 75, no. 2, pp. 299-304, 2013, DOI: $10.1007 / \mathrm{s} 10470-013-0049-3$. 